\title{
Systematic Review of Available Guidelines on Fertility Preservation of Young Patients with Breast Cancer
}

\author{
Mahnaz Haddadi ${ }^{1}$, Samad Muhammadnejad ${ }^{2}$, Fariba Sadeghi-Fazel ${ }^{3}$, Zahra \\ Zandieh $^{4}$, Gohar Rahimi ${ }^{5}$, Sanambar Sadighi ${ }^{6}$, Parya Akbari ${ }^{7}$, Mohammad-Ali \\ Mohagheghi $^{6}$, Alireza Mosavi-Jarrahi ${ }^{6,8}$, Saeid Amanpour ${ }^{1,9 *}$
}

\begin{abstract}
Background: Since the survival rate of breast cancer patients has improved, harmful effects of new treatment modalities on fertility of the young breast cancer patients has become a focus of attention. This study aimed to systematically review and critically appraise all available guidelines for fertility preservation in young breast cancer patients. Materials and Methods: Major citation databases were searched for treatment guidelines. Experts from relevant disciplines appraised the available guidelines. The AGREE II Instrument that includes 23 criteria in seven domains (scope and purpose of the guidelines, stakeholder involvement, rigor of development, clarity, applicability, editorial independence, and overall quality) was used to apprise and score the guidelines. Results: The search strategy retrieved 2,606 citations; 72 were considered for full-text screening and seven guidelines were included in the study. There was variability in the scores assigned to different domains among the guidelines. ASCO (2013), with an overall score of $\mathbf{6 8 . 0 \%}$, had the highest score, and St Gallen, with an overall score of $24.7 \%$, had the lowest scores among the guidelines. Conclusions: With the promising survival rate among breast cancer patients, more attention should be given to include specific fertility preservation recommendations for young breast cancer patients.
\end{abstract}

Keywords: Fertility preservation - breast cancer - young women - practice guidelines

Asian Pac J Cancer Prev, 16 (3), 1057-1062

\section{Introduction}

Breast cancer $(\mathrm{BC})$ is the most common malignancy diagnosed in women (Jemal et al., 2011; Bray et al., 2013). It is also the most frequent cancer in young women of reproductive age and comprises approximately $40 \%$ of all female cancers in this age group ( Jemal et al., 2009). Nearly $2 \%$ of BC is diagnosed in young women between 20 and 34 years of age, and $11 \%$ between 35 and 44 years of age ( Rodriguez-Wallberg and Oktay, 2010). The incidence of breast cancer in young women of reproductive age has been increasing in recent decades (Leclere et al., 2013; Keramatinia et al., 2014). Early diagnosis, adjuvant therapy, and higher cure rates have resulted in longer survival and lower mortality. It is estimated that a 50\% increase in the number of patients diagnosed with cancer will occur by 2030 . Currently, the five-year survival rate for BC is $>90 \%$ in developed countries. BC survivors are a major part of cancer survivors in many countries.
Young women with breast cancer are faced with many undesired consequences of cancer therapies, such as long duration of treatment, psychosocial problems, infertility, sexual dysfunction, and even occurrence of other cancers. For these reasons, quality of life remains an important consideration for patients surviving BC, particularly those $<40$ years of age, and interdisciplinary collaboration has become an essential part of survivor management. Among various side effects of $\mathrm{BC}$, the issues of fertility have been recognized with great importance for young women diagnosed with breast cancer (Sonmezer and Oktay, 2004; Ewertz and Jensen, 2010; Rodriguez-Wallberg and Oktay, 2010; Christinat and Pagani, 2012; Husseinzadeh and Husseinzadeh, 2013; Lange et al., 2013). After the publication of first guidelines on fertility preservation (FP) for cancer patients by American Society of Clinical Oncology (ASCO) in 2006 (Lee et al., 2006), and National Institute for Health and Clinical Excellence (NICE) in 2004 (National Collaborating Center for Women's

${ }^{1}$ Cancer Models Research Center, Cancer Institute of Iran, ${ }^{2}$ Research Center for Molecular and Cellular Imaging, ${ }^{6}$ Cancer Research Center, Cancer Institute of Iran, ${ }^{7}$ School of Medicine, ${ }^{9}$ IVF-Laboratory, Vali-e-Asr Reproductive Health Research Center, Tehran University of Medical Sciences, ${ }^{4}$ Anatomy Department, School of Medicine, Iran University of Medical Sciences, Tehran, ${ }^{3}$ Health Management Department, Quality Assurance Deputy, Razi Vaccine \& Serum Research Institute, Karaj, Iran, ${ }^{5}$ Research Group for Reproductive Medicine and IVF-Laboratory, Department of Obstetrics and Gynecology, Cologne University, Cologne, Germany, ${ }^{8}$ Faculty of Health Sciences, Simon Fraser University, Burnaby, BC, Canada *For correspondence: amanpour_s@tums.ac.ir 
and Children's Health (UK), 2004), oncologists are recommended to discuss the influences of cancer therapies on fertility with their young patients as early as possible and consider fertility preservation as a part of care. Both of these guidelines were updated recently (Fields et al., 2013; Loren et al., 2013). As a result of considerable improvement in assisted reproductive technology (ART), nowadays there are several options to restore fertility in young women including cryopreservation of oocyte, embryo and ovarian tissue, and in vitro maturation of oocyte before starting cancer treatment. Except embryo freezing, most of these techniques were investigational during the release of the first ASCO and NICE guidelines. Cryopreservation of ovarian tissue seems to be a promising method of preserving fertility in the young breast cancer patients (YBCP). This method is also so important for the patient's physiology that can avoid menopausal symptoms. Ovarian freezing as the emerging discipline of assisted reproductive technologies is progressively attracting interest to preserve fertility for young cancer patients. Fertility preservation options have also been used for other diseases such as lupus, glomerulonephritis, myelodysplasia, and premature ovarian failure as well as in women who wish to preserve their ovarian function for future reproductive potential and delayed childbearing age (Posada et al., 2001; Imhof et al., 2004; Demeestere et al., 2007; Maltaris et al., 2007; Isachenko et al., 2007; Ajala et al., 2010; Rahimi et al., 2010; Dolmans et al., 2010; Michaeli et al., 2012; Husseinzadeh, 2013). Despite rapid progress in cryopreservation technology and existing other resources and infrastructure, fertility preservation for young breast cancer patients is in its early stage with many challenges

(Posada et al., 2001; Imhof et al., 2004; National Collaborating Center for Women's and Children's Health (UK), 2004; Sonmezer and Oktay, 2004; Lee et al., 2006; Demeestere et al., 2007; Isachenko et al., 2007; Maltaris et al., 2007; Ajala et al., 2010; Dolmans et al., 2010; Ewertz and Jensen, 2010; Rahimi et al., 2010; Christinat and Pagani, 2012; Klemp and Kim, 2012; King et al., 2012; Michaeli et al., 2012; Klemp and Kim, 2012; King et al., 2012; Fields et al., 2013; Husseinzadeh, 2013; Husseinzadeh and Husseinzadeh, 2013; Lange et al., 2013; Leclere et al., 2013; Loren et al., 2013; Ronn and Holzer, 2013). The present study aimed to systematically review available guidelines on preservation of fertility in breast cancer patients.

\section{Materials and Methods}

\section{Systematic literature search}

A systematic literature search for existing guidelines of FP in patients with BC was performed using MEDLINE and Google Scholar databases. The search strategy comprised three main components: guidelines in any terms, breast cancer in appropriate terms, and fertility preservation in any possible terms (Appendix 1). In addition, eight Guideline Websites were searched including National Guideline Clearing House (http://www.guideline.gov/), National Comprehensive Cancer Network (http://www. nccn.org/professionals/physician_gls/f_guidelines.asp),
The Scottish Intercollegiate Guidelines Network (http:// www.sign.ac.uk/), The Canadian Medical Association InfoBase for Clinical Practice Guidelines (http://www. cma.ca/cpgs/), Guidelines International Networks (http://www.g-i-n.net/), American College of Physicians Clinical Practice Guidelines (http://www.acponline. org/clinical_information/guidelines/guidelines/), NICE (http://www.nice.org.uk/), and Fertile Hope (http://www. fertilehope.org). Searches were limited to papers published in English language from December 2003 to December 2013. Guidelines to fertility preservation in patients with breast cancer were included using consensus or evidencebased strategies. The latest version was included if the guidelines had been updated.

\section{Quality and content evaluation}

The guideline appraisal group comprised four experts from disciplines of medical oncology, epidemiology, cancer surgery, and assisted-reproductive technology. The quality of guidelines was assessed by AGREE II Instrument (AGREE Collaboration, 2003), 1 in which 23 criteria in seven domains were evaluated. These include the scope and purpose of the guidelines, stakeholder involvement, and rigor of development, clarity, applicability, editorial independence, and overall quality. Each appraiser scored the guidelines independently, and results were gathered and analyzed by other investigators who did not participate in the assessment. The appraisers' scores were expressed as standardized domain scores on a percentage scale (0\%-100\%).

\section{Results}

The search strategy retrieved 2606 citations; 72 were considered for full-text screening, and seven guidelines (Goldhirsch et al., 2009; Von Wolff et al., 2011; Cardoso et al., 2012; Coccia et al., 2012; ISFP Practice Committee et al., 2012; Klemp and Kim, 2012; Loren et al., 2013) were included in the study (Figure 1).

The guidelines included were from the American Society of Clinical Oncology (ASCO 2013), European Society of Breast Cancer Specialists (EUSOMA 2012), International Society for Fertility Preservation (ISFP, May, 22 and 31 2012), National Comprehensive Cancer Network (NCCN 2012), FertiPROTEKT (2011), and St Gallen International Expert Consensus (2009). The ASCO

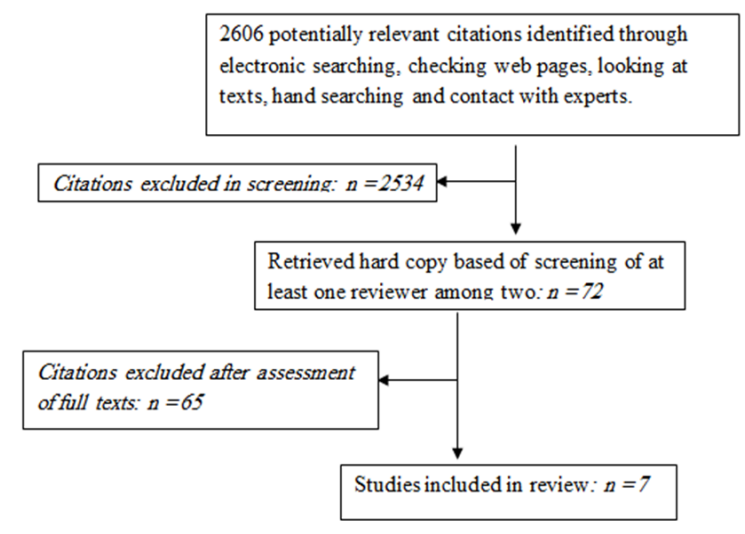

Figure 1. Quorum Diagram 
Table 1. AGREE II Domain Scores for Fertility Preservation Guidelines in Patients with Breast Cancer

\begin{tabular}{|c|c|c|c|c|c|c|c|}
\hline Organization [Year] & $\begin{array}{l}\text { Scope and } \\
\text { purpose } \%\end{array}$ & $\begin{array}{c}\text { Stakeholder } \\
\text { involvement } \%\end{array}$ & $\begin{array}{c}\text { Rigor of } \\
\text { development } \%\end{array}$ & $\begin{array}{c}\text { Clarity of } \\
\text { presentation } \%\end{array}$ & Applicability $\%$ & $\begin{array}{l}\% \quad \text { Editorial } \\
\text { independence } \%\end{array}$ & $\begin{array}{l}\text { Overall } \\
\text { score } \%\end{array}$ \\
\hline$\overline{\mathrm{ASCO} 14[2013]}$ & 69.4 & 91.7 & 62.5 & 50 & 71.9 & 62.5 & 68 \\
\hline EUSOMA30[2012] & 45.8 & 63.8 & 44.8 & 62.5 & 17.7 & 37.5 & 45.4 \\
\hline ISFP26[2012, specific] & 79.3 & 30.6 & 13 & 73.6 & 42.7 & 50 & 48.2 \\
\hline ISFP31[2012, general] & 45.8 & 48.6 & 6.3 & 79.2 & 15.6 & 50 & 42 \\
\hline NCCN32[2012] & 47.2 & 44.4 & 25 & 65.3 & 26 & 50 & 45.1 \\
\hline FertiPROTEKT33[2011] & 55.6 & 61.1 & 40.6 & 91.6 & 44.8 & 50 & 57.3 \\
\hline St Gallen34[2009] & 25 & 43.1 & 18.8 & 30.1 & 18.8 & 12.5 & 24.7 \\
\hline Mean score & 52.6 & 54.8 & 30.1 & 64.6 & 33.9 & 44.6 & 47.2 \\
\hline
\end{tabular}

Notes: ASCO=American Society of Clinical Oncology; EUSOMA=European Society of Breast Cancer Specialists; ISFP=International Society for Fertility Preservation; NCCN=National Comprehensive Cancer Network

Table 2. Fertility Preservation Measures Recommended by Guidelines

\begin{tabular}{|c|c|c|c|c|c|c|c|}
\hline & $\begin{array}{l}\text { Embryo } \\
\text { freezing }\end{array}$ & $\begin{array}{l}\text { Egg } \\
\text { freezing }\end{array}$ & $\begin{array}{l}\text { Ovarian tissue } \\
\text { freezing }\end{array}$ & $\begin{array}{c}\text { Ovarian } \\
\text { suppression }\end{array}$ & Donor eggs & Surrogacy & Adoption \\
\hline ASCO14[2013] & $\checkmark$ & $\checkmark$ & $\checkmark$ & $\checkmark$ & $\checkmark$ & $\checkmark$ & $\checkmark$ \\
\hline EUSOMA30[2012] & $\checkmark$ & $\checkmark$ & $\checkmark$ & $\checkmark$ & $x$ & $x$ & $x$ \\
\hline ISFP26[2012, specific] & $\checkmark$ & $\checkmark$ & $\checkmark$ & $\checkmark$ & $x$ & $x$ & $x$ \\
\hline ISFP31[2012, general] & $\checkmark$ & $\checkmark$ & $\checkmark$ & $\checkmark$ & $x$ & $x$ & $x$ \\
\hline NCCN32[2012] & $\checkmark$ & $\checkmark$ & $\checkmark$ & $\checkmark$ & $x$ & $x$ & $x$ \\
\hline FertiPROTEKT33[2011] & $\checkmark$ & $\checkmark$ & $\checkmark$ & $\checkmark$ & $x$ & $x$ & $x$ \\
\hline St Gallen34[2009] & $\checkmark$ & $\checkmark$ & $\checkmark$ & $x$ & $x$ & $x$ & $x$ \\
\hline
\end{tabular}

guideline was an updated version; the rest were new. ISFP provided two guidelines; one guideline was specifically related to breast cancer patients' fertility preservation. There was variability in the scores assigned to different domains between the guidelines. Among the different domains, the rigor of development had the lowest scores for all the guidelines compared with the other domains. ASCO (2013), with an overall score of $68.0 \%$, had the highest score, and St Gallen, with an overall score of $24.7 \%$, had the lowest scores among the guidelines. In the domain of scope and purpose, there were similarities in assigning scores to different guidelines, except St Gallen that scored very low (a score of $25.0 \%$ ). ASCO received the highest score $(91.7 \%)$ in the domain of stakeholder involvement, whereas the majority of guidelines performed $<50 \%$ in this domain. Many of the guidelines received scores of $>50 \%$ in clarity of presentation, except St. Gallen, with a low score of $30.1 \%$. Except for ASCO that scored high $(71.9 \%)$, other guidelines scored $<50 \%$ in the applicability domain. In the editorial independency domain, EUSOMA had a score of $37.5 \%$ and St. Gallen had a score of $12.5 \%$, whereas other guidelines had scores $>50 \%$. The ASCO and NCCN guidelines described the guideline development process and stakeholder involvement in detail, whereas the remaining guidelines provided some brief description without details of stakeholder involvement. With the exception of ASCO, none of the guidelines reported a systematic search strategy in their methodology section. The detailed AGREE II appraisal results are presented at Table 1.

Only one guideline (ISFP) was exclusively devoted to fertility preservation for young patients with breast cancer, whereas the others recommend fertility preservation for other cancers. Seven fertility preservation procedures recommended for patients were identified across the guidelines (Table 2). Embryo freezing, mature oocyte cryopreservation and ovarian tissue freezing were recommended by all the guidelines. Ovarian suppression using GnRH agonists before chemotherapy were advised by six guidelines (Table 2). Donor eggs, surrogacy, and adoption were proposed only by ASCO.

\section{Discussion}

Our study systematically reviewed fertility preservation using major guidelines. The importance of fertility preservation in young breast cancer patients was assessed in the major practicing and currently used guidelines. All the evaluated guidelines had recommendations on fertility preservation for cancer patients undergoing chemotherapy or other treatment modalities with a high risk of infertility.

It is obvious that fertility preservation is an important issue for reproductive-age breast cancer patients. ASCO, NICE, American Society for Reproductive Medicine, and other important organizations recommend that oncology centers should offer fertility counseling to their patients (Goldhirsch, et al., 2009; Pentheroudakis, et al., 2010; Von Wolff, et al., 2011;Klemp and Kim, 2012; Cardoso, et al., 2012; ISFP Practice Committee1, et al., 2012; Fields, et al., 2013; Loren, et al., 2013). Young women represent a relatively small proportion of breast cancer patients and their options for fertility preservation have been limited by many factors such as safety, efficacy issues, and inadequate time before starting cancer therapies. These women experience strong psychosocial distress and also face long-term impairments in their quality of life and fertility. Additionally, the problems of young patients affect some of their relatives, and more people come to bear the breast cancer burden (Sonmezer and Oktay, 2004; Cruz et al., 2010; ISFP Practice Committee1 et al., 2012;). Studies has reported gaps in young cancer patients receiving fertility counseling even in the developed 
countries for a simple reason that a young age at diagnosis reserve enough in future reproductive time ( Ronn and Holzer, 2013; Reinecke, 2013). It is estimated that even at the best and most favorable scenario, undergoing antihormonal treatment alone can delay pregnancy for at least 5 years. Chemotherapy may cause severe follicle depletion, resulting in a loss of $\geq 10$ years in reproductive function. Conversely, progressive neoadjuvant therapy before surgery is completed in some of these patients with clinically positive nodes or larger tumor size $(>2$ $\mathrm{cm})$. Neoadjuvant therapy impairs fertility as well as limits the fertility preservation options. Most cases of invasive BC occur between 30 and 40 years of age, shedding light on the critical role of age in diagnoses for desirable fertility preservation. In healthy females, after the age of 37-38 years, $>90 \%$ of oocytes present at birth have already undergone atresia. Therefore, there are differences between the characterization of a young woman in oncology and gynecology (Kim et al., 2011; Lobo, 2005; Peccatori et al., 2012). The age-related fertility preservation options are not completely clear in current guidelines for oncologists, and may be very important because the value of early fertility preservation counseling for young cancer patient fully depends on it. Rapid counseling may allow YBCP sufficient time for one or two rounds of egg collection without delaying the start of their cancer treatmeKim et al., 2011). Some investigators have reported that the benefit of $\mathrm{GnRH}$ agonist therapy to protection of ovaries is unproven and it should not be offered as individual method of fertility preservation in young breast cancer patient (Sonmezer and Oktay, 2006; Bedoschi et al., 2013). Recent studies suggest that GnRH with chemotherapy in premenopausal women is associated with higher rates of resumption of menses, although it is not associated with improvement in pregnancy rates. Usage of less gonadotoxic regimens for adjuvant or neoadjuvant chemotherapy is a good choice and may be considered in YBCP with favorable tumors characteristic (Lobo, 2005; Kim et al., 2011). When cryopreservation of embryos or oocytes is not possible, ovarian tissue cryopreservation can be considered without a delay in cancer treatment (Oktay et al., 2003; 2005; Dolmans et al., 2010; Rahimi et al., 2010; Kim et al., 2011; ISFP Practice Committee et al., 2012; Husseinzadeh, 2013). Although many scientific or technical aspects of fertility preservation options are presented in current recommendations and guidelines, this matter raises several important ethical and legal issues that should be adequately addressed to young patients or parents before such techniques are used. Any decision must be in the patient's preference and best interest. A very informed consent to accept any fertility preservation options is both a legal and an ethical need that must take consideration in practice ( Ethics Committee of the American Society for Reproductive Medicine, 2005; Wallace et al., 2005; Jeruss and Woodruff, 2009; Klock et al., 2010). Fertility preservation in cancer patients is an interdisciplinary approach. It needs the simultaneous functions of many components, which is a challenge. Many studies have shown that the most important weak points are poor access and referral to fertility services, low level of knowledge, and lack of available education for patients and health care providers (Forman et al., 2010; Klemp and Kim, 2012; King et al., 2012; Ronn and Holzer, 2013; Peate et al., 2013; Lange et al., 2013). Fertility preservation in breast cancer patients is even more complicated in underdeveloped countries with unsatisfactory health care infrastructure. Breast cancer survivors are one of the largest portion of cancer survivors (Dizon, 2009). Our results and many other reports demonstrate that comprehensive, specific, and practical guidelines for young breast cancer patients' fertility preservation are rare (Ethics Committee of the American Society for Reproductive Medicine, 2005; Von Wolff et al., 2011). The lack of a suitable guideline that covers all aspects of fertility preservation in young patients with breast cancer is evident. An appropriate guideline for YBCP needs to offer fertility preservation options, considering costs, practicality, social values, and ethical consequences. Such a guideline should consider establishment of a multidisciplinary approach, providing effective engagement between oncologists, fertility specialists, and patients. Fertility preservation should be routinely started as early as possible after the diagnosis of breast cancer. Such a system also helps to decrease the pressure of decision making for both the patient and the oncologist.

In conclusion, in this study, we retrieved seven guidelines of fertility preservation for breast cancer patients. Critical appraisal of the present guidelines indicates that these guidelines do not appropriately address all aspects of a comprehensive guideline development. Not all available guidelines address specific recommendations for fertility preservation in young breast cancer patients. With the promising survival rate among breast cancer patients, more attention should be given to specific fertility preservation recommendations in young breast cancer patients.

\section{Acknowledgements}

The authors express their gratitude to Dr. Kazem Zendehdel and Dr. Bahman Mazheri who supported the development of this work. This work was supported with a grant from the Cancer Models Research Center, Tehran University of Medical Sciences, Tehran, Iran.

\section{References}

AGREE Collaboration (2003). Development and validation of an international appraisal instrument for assessing the quality of clinical practice guidelines: the AGREE project. Qual Saf Health Care, 12, 18-23.

Ajala T, Rafi J, Larsen-Disney P, et al (2010). Fertility preservation for cancer patients: a review. Obstet Gynecol Int, 2010, 160386.

Bedoschi G, Turan V, Oktay K (2013). Utility of GnRH-agonists for fertility preservation in women with operable breast cancer: is it protective?. Curr Breast Cancer Rep, 5, 302-8.

Bray F, Ren JS, Masuyer E, et al (2013). Global estimates of cancer prevalence for 27 sites in the adult population in 2008. Int J Cancer, 132, 1133-45.

Cardoso F1, Loibl S, Pagani O, et al (2012). The European 
Society of Breast Cancer Specialists recommendations for the management of young women with breast cancer. Eur J Cancer, 48, 3355-77.

Christinat A, Pagani O (2012). Fertility after breast cancer. Maturitas, 73, 191-6.

Coccia PF, Altman J, Bhatia S, et al (2012). Adolescent and young adult oncology. JNCCN, 10, 1112-50.

Cruz MR, Prestes JC, Gimenes DL, et al (2010). Fertility preservation in women with breast cancer undergoing adjuvant chemotherapy: a systematic review. Fertil Steril, 94, 138-43.

Demeestere I, Simon P, Emiliani S, et al (2007). Fertility preservation: successful transplantation of cryopreserved ovarian tissue in a young patient previously treated for Hodgkin's disease. Oncologist, 12, 1437-42.

Dizon DS (2009). Quality of life after breast cancer: survivorship and sexuality. Breast $J, \mathbf{1 5}, 500-4$.

Dolmans MM, Jadoul P, Gilliaux S, et al (2013). A review of 15 years of ovarian tissue bank activities. J Assist Reprod Genet, 30, 305-14.

Ethics Committee of the American Society for Reproductive Medicine (2005). Fertility preservation and reproduction in cancer patients. Fertil Steril, 83, 1622-8.

Ewertz M, Jensen AB (2010). Late effects of breast cancer treatment and potentials for rehabilitation. Acta Oncol, 50, $187-93$.

Fields E, Chard J, James D, et al (2013). Fertility [update]: summary of NICE guidance. BMJ, 20, 346-650.

Forman EJ, Anders CK, Behera MA (2010). A nationwide survey of oncologists regarding treatment-related infertility and fertility preservation in female cancer patients. Fertil Steril, 94, 1652-6.

Goldhirsch A, Ingle JN, Gelber RD, et al (2009). Thresholds for therapies: highlights of the St Gallen International Expert Consensus on the primary therapy of early breast cancer 2009. Ann Oncol, 20, 1319-29.

Husseinzadeh N (2013). Preservation of ovarian function in young women with gynecologic cancer desiring future pregnancy: a review. AJCR, 1, 25-37.

Husseinzadeh N, Husseinzadeh HD (2013). Preservation of fertility in female cancer patients desiring future child bearing; what is available and what can be offered. World J Oncol, 4, 1-7.

Imhof M, Hofstetter G, Bergmeister 1H, et al (2004). Cryopreservation of a whole ovary as a strategy for restoring ovarian function. J Assist Reprod Genet, 21, 459-65.

Isachenko V, Isachenko E, Reinsberg J ,et al (2007). Cryopreservation of human ovarian tissue: comparison of rapid and conventional freezing. Cryobiology, 55, 261-8.

ISFP Practice Committee1, Kim SS, Donnez J, et al (2012). Recommendations for fertility preservation in patients with lymphoma, leukemia, and breast cancer. J Assist Reprod Genet, 29, 465-8.

Jemal A, Bray F, Center MM, et al (2011). Global cancer statistics. CA Cancer J Clin, 61, 69-90.

Jemal CK, Johnson R, Litton J, et al (2009). Breast cancer before age 40 years. Semin Oncol, 36, 237-49.

Jeruss JS, Woodruff TK (2009). Preservation of fertility in patients with cancer. $N$ Engl J Med, 360, 902-11.

Keramatinia A, Mousavi-Jarrahi SH, Hiteh M, et al (2014). Trends in incidence of breast cancer among women under 40 in Asia. Asian Pac J Cancer Prev, 15, 1387-90.

Kim SS, Klemp J, Fabian C (2011). Breast cancer and fertility preservation. Fertil Steril, 95, 1535-43.

King JW, Davies MC, Roche N, et al (2012). Fertility preservation in women undergoing treatment for breast cancer in the UK: a questionnaire study. Oncologist, 17, 910-16.
Klemp JR, and Kim SS (2012). Fertility preservation in young women with breast cancer. J Assist Reprod Genet, 29, 469-72.

Klock SC, Zhang JX, Kazer RR (2010). Fertility preservation for female cancer patients: early clinical experience. Fertil Steril, 94, 149-55.

Lange S, Hurst BS, Matthews ML, et al (2013). Fertility preservation in patients with gynecologic cancer-part I: the impact of gynecologic malignancies on fertility. Postgraduate Obstet Gynecol, 33, 1-7.

Lange S, Hurst BS, Matthews ML, et al (2013). Fertility preservation in patients with gynecologic cancer--part ii: current options and the ethics of oncofertility. Postgraduate Obstet Gynecol, 33, 1-5.

Leclere B1, Molinie F, Tretarre B, et al (2013). Trends in incidence of breast cancer among women under 40 in seven European countries: a GRELL cooperative study. Cancer Epidemiol, 37, 544-9.

Lee SJ, Schover LR, Partridge AH, et al (2006). American society of clinical oncology recommendations on fertility preservation in cancer patients. J Clin Onco, 24, 2917-31.

Lobo RA (2005). Potential options for preservation of fertility in women. $N$ Engl J Med, 353, 64-73.

Loren AW, Mangu PB, Beck LN, et al (2013). Fertility preservation for patients with cancer: American Society of Clinical Oncology clinical practice guideline update. J Clin Oncol, 31, 2500-10.

Maltaris T, Seufert R, Fischl F, et al (2007). The effect of cancer treatment on female fertility and strategies for preserving fertility. Eur J Obstet Gynecol Reprod Biol, 130, 148-55.

Michaeli J, Weintraub M, Gross E, et al (2012). Fertility preservation in girls. Obstet Gynecol Int, 2012, 139193.

National Collaborating Center for Women's and Children's Health (UK) (2004). Fertility: Assessment and Treatment for People with Fertility Problems. RCOG Press.

Oktay K, Buyuk E, Davis O, et al (2003). Fertility preservation in breast cancer patients: IVF and embryo cryopreservation after ovarian stimulation with tamoxifen. Hum Reprod, 18, 90-5.

Oktay K, Buyuk E, Libertella N, et al (2005). Fertility preservation in breast cancer patients: a prospective controlled comparison of ovarian stimulation with tamoxifen and letrozole for embryo cryopreservation. J Clin Oncol, 23, 4347-53.

Partridge AH (2008). Fertility preservation: a vital survivorship issue for young women with breast cancer. J Clin Oncol, 26, 2612-13.

Peate M, Meiser B, Friedlander M, et al (2011). It's now or never: fertility-related knowledge, decision-making preferences, and treatment intentions in young women with breast cancer-an Australian fertility decision aid collaborative group study. J Clin Oncol, 29, 1670-77.

Peccatori FA, Pup LD, Salvagno F, et al (2012). Fertility preservation methods in breast cancer. Breast Care, $\mathbf{7}$, 197-202.

Pentheroudakis G, Orecchia R, Hoekstra HJ, et al (2010). Cancer, fertility and pregnancy: ESMO Clinical Practice Guidelines for diagnosis, treatment and follow-up. Ann Oncol, 21, 266-73.

Posada MN, Kolp L, García JE (2001). Fertility options for female cancer patients: facts and fiction. Fertil Steril, $\mathbf{7 5}$, 647-53.

Rahimi G, Isachenko V, Kreienberg R, et al (2010). Revascularisation in human ovarian tissue after conventional freezing or vitrification and xenotransplantation. Eur $J$ Obstet Gynecol Reprod Biol, 149, 63-7.

Reinecke J (2013). On the frontlines of cancer \& fertility: A 
Mahnaz Haddadi et al

Report on the 2013 ASRM Meeting. [www.asram.org]. LIVESTRONG.

Rodriguez-Wallberg KA, Oktay K (2010). Fertility preservation in women with breast cancer. Clin Obstet Gynecol, 53, 753-62.

Ronn R, and Holzer H (2013). Oncofertility in Canada: an overview of Canadian practice and suggested action plan. Curr Oncol, 20, 465-74.

Sonmezer M, Oktay K (2004). Fertility preservation in female patients. Hum Reprod Update, 10, 251-66.

Sonmezer M, Oktay K (2006). Fertility preservation in young women undergoing breast cancer therapy. Oncologist, 11, 422-34.

Theriault RL, Carlson RW, Allred C, et al (2013). Breast cancer, version 3.2013: featured updates to the NCCN guidelines. $J$ Natl Compr Canc Netw, 11, 753-60.

Von Wolff M, Dittrich R, Denschlag D, et al (2011). Fertility preservation in women--a practical guide to preservation techniques and therapeutic strategies in breast cancer, Hodgkin's lymphoma and borderline ovarian tumours by the fertility preservation network FertiPROTEKT. Arch Gynecol Obstet, 284, 427-35.

Wallace WH, Anderson RA, Irvine DS (2005). Fertility preservation for young patients with cancer: who is at risk and what can be offered?. Lancet Oncol, 6, 209-18. 